\title{
Long-Term Safety and Efficacy of Blonanserin Transdermal Patches in Japanese Patients with Schizophrenia: A 52-Week Open-Label, Multicenter Study
}

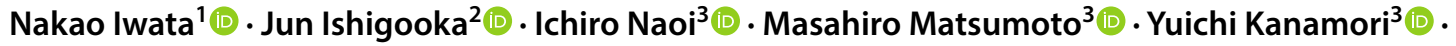 \\ Hiroshi Nakamura ${ }^{3}\left(\mathbb{D} \cdot\right.$ Teruhiko Higuchi $^{4}(\mathbb{D}$
}

Published online: 27 December 2019

(c) The Author(s) 2019

\begin{abstract}
Background Blonanserin transdermal patch therapy is now available in Japan for the treatment of schizophrenia and may provide several advantages over the tablet formulation.

Objective The aim was to evaluate the long-term safety and efficacy of blonanserin transdermal patches in Japanese patients with schizophrenia.

Methods An open-label study was conducted in adults with schizophrenia at 37 sites in Japan. Patients were enrolled in either cohort 1 or 2 . Patients in cohort 1 received $8-16 \mathrm{mg} /$ day blonanserin tablets for 6 weeks and then $40-80 \mathrm{mg} / \mathrm{day}$ blonanserin patches for 52 weeks. The dose of blonanserin patches was determined according to the dose of the tablets. In cohort 2, every patient started from $40 \mathrm{mg} /$ day and then $40-80 \mathrm{mg} /$ day blonanserin transdermal patches for 52 weeks. Both cohorts had 1-2 weeks of follow-up. Safety endpoints included the incidence of adverse events (AEs), treatment-related AEs, extrapyramidal AEs [also assessed using the change in Drug-Induced Extrapyramidal Symptoms Scale (DIEPSS) score], the use of any concomitant antiparkinsonian drugs, and skin-related AEs, including skin irritation. Patients also underwent assessment of laboratory values including for serum prolactin concentration, vital signs, body weight, electrocardiographic (ECG) changes, and the corrected QT (QTc) interval. Suicidal ideation was assessed via the Columbia-Suicide Severity Rating Scale (C-SSRS) score. Efficacy was assessed via duration of blonanserin transdermal patch treatment, Positive and Negative Syndrome Scale (PANSS) total and subscale scores, and Clinical Global Impression-Severity (CGI-S) scores. Other endpoints included total Drug Attitude Inventory 10 (DAI-10) scores, EuroQol-5 Dimension (EQ-5D) effect values, and a patient questionnaire about the dosage form.

Results A total of 223 patients with consents, 117 in cohort 1 and 106 in cohort 2 were included in the study. Of the 117 patients in cohort 1, 108 were treated with blonanserin tablets, and 97 received blonanserin patches and were included in the safety analysis set. In cohort 2, 103 of the 106 patients were treated with blonanserin transdermal patches and were included in the safety analysis set. In total, 91 patients were male (45.5\%). The mean age was 43.8 years. Discontinuation occurred in 40 patients $(41.2 \%)$ in cohort 1 and 44 patients $(42.7 \%$ ) in cohort 2. Discontinuation resulted from AEs in $18.6 \%$ (cohort 1 ) and $11.7 \%$ (cohort 2) and from withdrawal of consent in $13.4 \%$ (cohort 1) and 20.4\% (cohort 2), and seven patients overall discontinued due to skin reactions. AEs were reported in 174 patients (87.0\%), and 13 serious AEs occurred in 12 patients (6.0\%), of which six patients were in cohort 1 and six patients were in cohort 2. Serious AEs were six schizophrenia $(n=6)$ and seven other AEs $(n=6)$, which included impulse-control disorder, fracture, epistaxis, asthma, pneumonia aspiration, pneumonia hemophilus, and pneumonia. The most common AEs were nasopharyngitis $(n=62,31.0 \%)$, application site erythema $(n=45,22.5 \%)$, application site pruritus $(n=23,11.5 \%)$, and akathisia $(n=20,10.0 \%)$. AE incidence was similar in cohort $1(84.5 \%)$ and cohort 2 (89.3\%). Extrapyramidal and skin-related AEs were reported in 51 patients (25.5\%) and 83 patients (41.5\%), respectively. None of these AEs were serious. The mean change from baseline in total DIEPSS score
\end{abstract}

Electronic supplementary material The online version of this article (https://doi.org/10.1007/s40263-019-00692-6) contains supplementary material, which is available to authorized users.

Extended author information available on the last page of the article 
at Week 52 \{last observation carried forward [LOCF] (standard deviation [SD])\} was -0.1 (1.55), indicating no marked effect. In terms of concomitant medications used in cohort 1 and cohort 2, 33.0\% (32/97) and 22.3\% (23/103) used antiparkinsonian drugs, respectively. The majority of skin-related AEs occurred early in treatment and were appropriately managed with topical therapies. Of the patients who answered "No" to all C-SSRS categories at baseline $(n=129), 13$ patients $(10.1 \%)$ were evaluated as having emergence of suicidal ideation. Among patients who answered "No" to all C-SSRS suicidal behavior categories at baseline $(n=172)$, one $(0.6 \%)$ was evaluated as having suicidal behavior during blonanserin transdermal patch treatment. There were no clinically significant changes in laboratory tests or examinations, including prolactin level, vital signs, body weight, ECG, metabolism-related parameters, and QTc interval. The mean (SD) change in body weight was -0.04 (4.561) $\mathrm{kg}$ and -0.67 (6.841) $\mathrm{kg}$ in cohort 1 and cohort 2, respectively. The mean changes from baseline in PANSS total score at Week 52 (LOCF [SD]) were -0.1 [11.6] and - 3.4 [15.3] in cohort 1 and 2, respectively. PANSS scores did not change after switching from tablet to patch formulation in cohort 1 and decreased over the 52 weeks of treatment with the blonanserin patches. The mean change from baseline in CGI-S score at Week 52 (LOCF [SD]) was - 0.2 [1.03] in both cohorts combined. After 52 weeks of blonanserin patch treatment, the total DAI-10 score increased or remained unchanged compared with baseline in 82 of the 129 patients (63.6\%) for whom these data were available. In the intention-to-treat population of the combined cohorts $(n=200)$, the mean (SD) change from baseline in EQ-5D score at the last assessment was -0.0365 (0.17603). Patients' attitudes to the blonanserin transdermal patches were generally positive.

Conclusions Blonanserin transdermal patches are safe and effective in the long-term treatment of schizophrenia.

ClinicalTrials.gov registration NCT02335658.

\section{Key Points}

Blonanserin transdermal patches were well tolerated over 52 weeks and showed maintained efficacy in patients with schizophrenia.

Blonanserin patches had a long-term safety profile similar to, or better than, that of blonanserin tablets; skinrelated adverse events were all mild or moderate and mostly occurred early in treatment.

Long-term blonanserin transdermal patch therapy was associated with stabilization of both the positive and negative symptoms of schizophrenia, and patients' attitudes towards blonanserin transdermal patches were generally positive.

\section{Introduction}

Blonanserin is a second-generation antipsychotic (SGA) that is approved for the treatment of schizophrenia in Japan, South Korea, and China [1, 2]. It has strong affinity for $\mathrm{D}_{2}$ and $\mathrm{D}_{3}$ dopamine and 5-HT $2 \mathrm{~A}$ serotonin receptors, where it exerts antagonist activity [1]. Blonanserin has low or very low affinity for other neurotransmitter receptor subtypes (dopamine $\mathrm{D}_{1}, \mathrm{D}_{4}$, and $\mathrm{D}_{5}$, adrenoceptors $\alpha_{1}, \alpha_{2}$, and $\beta$, serotonin $5-\mathrm{HT}_{1 \mathrm{~A}}, 5-\mathrm{HT}_{2 \mathrm{~B}}$, and 5- $\mathrm{HT}_{2 \mathrm{C}}$, histamine $\mathrm{H}_{1}$, and muscarinic $\mathrm{M}_{1}$ and $\mathrm{M}_{3}$ ) $[1,3]$. In short- and long-term studies, blonanserin was effective and well tolerated in patients with schizophrenia [2, 4-9]. In a meta-analysis of randomized controlled clinical trials, blonanserin was more effective than aripiprazole in improving total Positive and Negative Syndrome Scale (PANSS) scores, and was associated with a lower risk of hyperprolactinemia, but a higher risk of akathisia, agitation/excitement, and extrapyramidal symptoms (EPS), compared with a combination of risperidone and paliperidone [10].

Non-adherence to drug therapy is a serious unresolved issue for schizophrenia treatment. A positive attitude toward medication is one of the key factors needed to improve adherence, as patients' attitude is significantly associated with treatment adherence [11]. Dosage form also influences adherence to long-term drug therapy [12]. Commonly used atypical antipsychotics for long-term treatment of schizophrenia are mainly available in tablet formulation. When poor adherence is found, long-acting injections are also used.

The addition of a new dosage form is expected to be helpful for patients with schizophrenia who prefer not to use the current dose formulations, and this may be useful in increasing treatment adherence. We considered the main advantages of a transdermal patch formulation as follows: easy supervision by the medical staff or caregiver, providing stable blood concentrations over long periods, and avoiding first-pass metabolism that can affect plasma levels of oral agents [13].

Blonanserin is available as transdermal patches $(20 \mathrm{mg}$ and $40 \mathrm{mg}$ ), which is expected to provide a number of advantages over the tablet formulation. For example, transdermal patches can provide stable blood concentrations over long periods and avoid first-pass metabolism that can affect plasma levels of oral agents [14]. This may improve adherence and decrease the incidence of adverse events (AEs) that occur as a result of high 
plasma concentrations immediately after administration. Of the long-acting antipsychotic formulations, transdermal systems have advantages over intramuscular injections because they are non-invasive and treatment can be stopped immediately, if needed, by removing the patch [14]. In a 6-week randomized, double-blind, multicenter, phase III study conducted in patients with acute-phase schizophrenia, blonanserin transdermal patches were well tolerated and significantly improved PANSS total scores compared with placebo [13].

The aim of this multicenter, open-label study was to evaluate the safety and efficacy of the blonanserin transdermal patches when used for 52 weeks in the treatment of patients with schizophrenia. The safety and efficacy of the blonanserin patches were also evaluated in patients who switched to the transdermal formulation from blonanserin tablets or other antipsychotic drugs.

\section{Methods}

\subsection{Study Design}

This 52-week multicenter, open-label study was conducted at 37 medical institutions in Japan from February 2015 to May 2017 (Clinical Study Registration No. NCT02335658).

Patients were eligible for inclusion if they were aged $\geq 18$ years and met the diagnostic criteria for schizophrenia outlined in the Diagnostic and Statistical Manual of Mental Disorders 5th edition (DSM-5). Premenopausal (fertile) women were required to have a negative pregnancy test result at the time of screening. All female patients had to agree to use appropriate contraceptives, as did all male patients with a potentially fertile sexual partner. For a full list of exclusion criteria, see supplementary Table S1 (see the electronic supplementary material [ESM]).

Eligible patients who provided written informed consent were enrolled in either cohort 1 (patients switched from blonanserin tablets to patch therapy) or cohort 2 (patients received continuous blonanserin patch therapy; Fig. 1), and then entered a dose reduction/observation period of $\leq 4$ weeks, during which their previous antipsychotic treatment was withdrawn. The duration of the dose reduction/observation period depended on the dose of previous antipsychotic therapy, which had to be reduced to $\leq 12 \mathrm{mg}$ haloperidol equivalents.

The objective of cohort 1 was to evaluate the safety and efficacy of the blonanserin patches in patients who received blonanserin tablets prior to the transdermal patches. Patients in cohort 1 received blonanserin tablets twice daily (after breakfast and dinner) for 6 weeks (tablet treatment phase), followed by blonanserin patches once daily for 52 weeks (patch treatment phase) and a post-treatment follow-up period of 1-2 weeks (Fig. 1a). During the tablet treatment period, the initial dosage of blonanserin tablets was $8 \mathrm{mg} /$ day, which was then adjusted according to predefined criteria (Table S2). At the end of the tablet treatment phase, patients could be receiving blonanserin tablets at a dosage of 8,12 , or $16 \mathrm{mg} /$ day. The starting dose of blonanserin patches was determined by the final dose of oral blonanserin, i.e., patients who had received blonanserin tablets 8,12 , and $16 \mathrm{mg} /$ day received 40,60 , and $80 \mathrm{mg} /$ day patches, respectively (Table S3). This procedure means that patients started blonanserin patches from the comparable dose of tablets, which is one of the common situations in clinical settings. Patients applied 40-80 mg blonanserin transdermal patches to their back, chest, or abdomen by combining 20-mg or 40-mg patches. Blonanserin transdermal patch doses could be adjusted in a range between $40 \mathrm{mg}$ and $80 \mathrm{mg}$ by following the predefined criteria (Table S2) during the patch treatment phase.

The objective of cohort 2 was to evaluate the safety and efficacy of the blonanserin transdermal patches in patients, including those who had previously received any antipsychotic treatments or had not received a treatment for schizophrenia before. Patients entering cohort 2 received open-label treatment with blonanserin transdermal patches for 52 weeks, followed by a post-treatment follow-up period of 1-2 weeks (Fig. 1b). The cohort 2 study did not include an "oral tablet run-in" phase. All patients in cohort 2 started blonanserin transdermal patches from $40 \mathrm{mg}$, which is the minimum dose expected to be effective from the phase 2 trial result [15]. The conditions of the blonanserin transdermal patch treatment period were the same as those in cohort 1 (Table S2). Patients were enrolled in cohort 1 in order of entry, and enrollment in cohort 2 began after completion of cohort 1 enrollment.

This study was conducted in compliance with the ethical principles stated in the Declaration of Helsinki and with the Good Clinical Practice (GCP) guidelines and other relevant Japanese regulations. The study protocol was approved by the institutional review boards of all participating institutions. Written informed consent was obtained from all patients (and their legal guardian if the patient was $<20$ years old or was hospitalized for medical care and protection) prior to the study.

\subsection{Outcome Assessments}

The safety endpoints included treatment-emergent AEs (TEAEs) and treatment-related AEs, extrapyramidal AEs (including the use of any concomitant antiparkinsonian drugs), skin-related AEs at the application site, and skin irritation assessment. TEAEs were defined as any untoward medical event that occurred during blonanserin transdermal patch use. Treatment-related AEs were defined as AEs for which a causal relation to blonanserin could not be ruled out. 

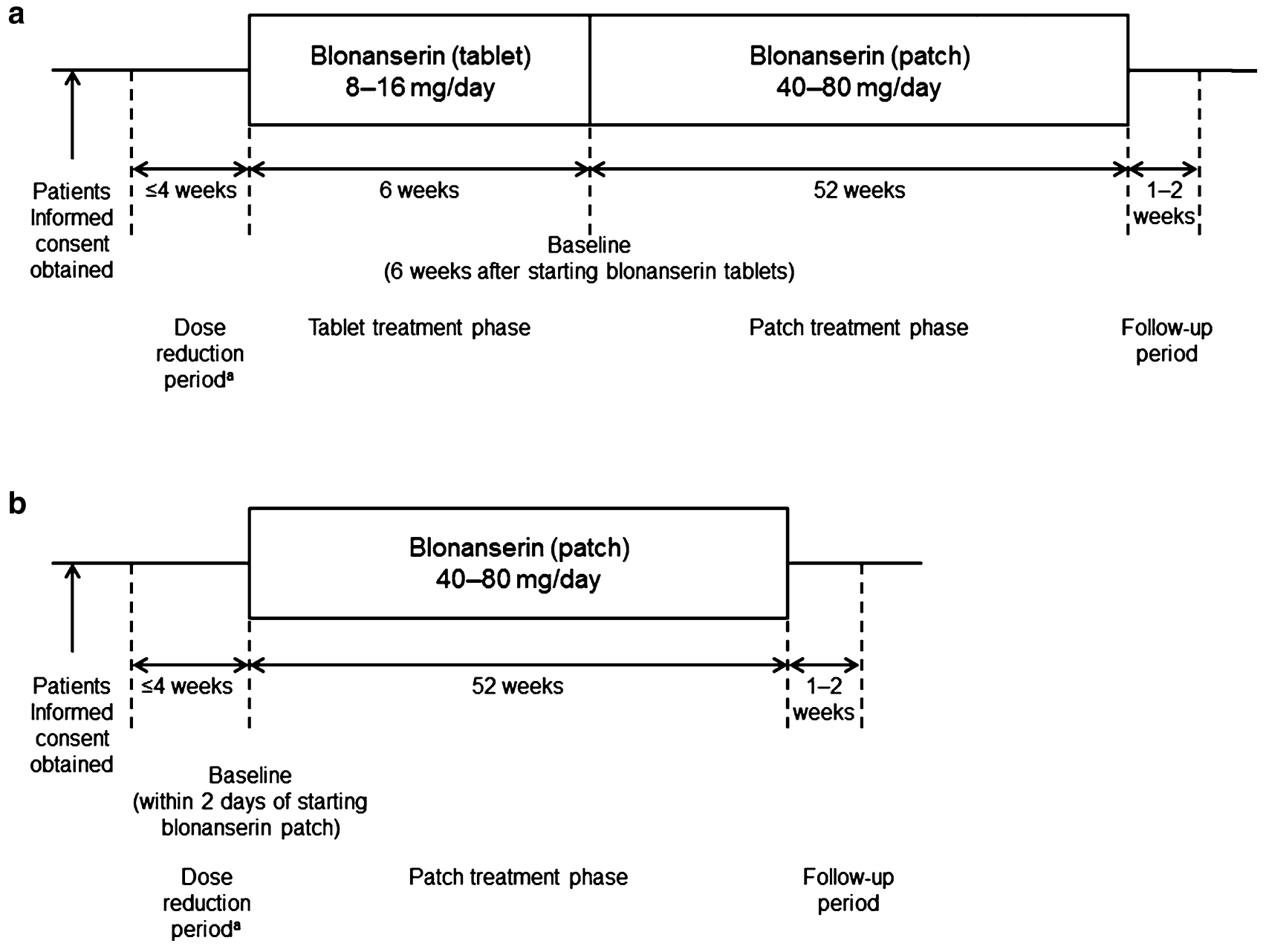

Fig. 1 Study design for a cohort 1 and $\mathbf{b}$ cohort 2. ${ }^{\text {a }}$ There was a dose reduction/observation period for patients who had been previously treated with antipsychotics at the dosage of $>12.0 \mathrm{mg} / \mathrm{day}$ in

AEs were recorded in accordance with the Medical Dictionary for Regulatory Activities (MedDRA) version 19.1. AEs that occurred more than once were counted only once in preferred term aggregation. Skin irritation was assessed on a 6-point scale (no reaction [-]; mild erythema [ \pm ]; erythema [+]; erythema/edema [++]; erythema/edema/papule, serous papule, vesicle $[+++]$; bulla $[++++])$ as described by Kawamura and colleagues [16]. The presence and severity of EPS were assessed using the change in Drug-Induced Extrapyramidal Symptoms Scale (DIEPSS) score [17] (total score not including overall severity and score for individual symptoms). Patients also underwent laboratory tests, including for serum prolactin concentration, and were assessed for vital signs, changes in body weight, and electrocardiographic (ECG) changes, specifically any changes in the corrected QT (QTc) interval. The potential for suicidal ideation was assessed using the Columbia-Suicide Severity Rating Scale (C-SSRS) score [18]. The development of suicidal haloperidol equivalents. Prior antipsychotics were first decreased to $\leq 12.0 \mathrm{mg} /$ day and then gradually discontinued

ideation or behavior was defined as patients who answered "No" to all the C-SSRS questions at baseline and answered "Yes" to any of the C-SSRS questions after starting blonanserin transdermal patch treatment.

Efficacy endpoints assessed in the present study included duration of blonanserin transdermal patch treatment, change in total PANSS score, change in total PANSS subscale score, change in total PANSS 5-factor score [19], and change in Clinical Global Impression-Severity (CGI-S) score from baseline to the end of the blonanserin transdermal patch treatment phase. Other endpoints included total Drug Attitude Inventory 10 (DAI-10) score [20] and EuroQol-5 Dimension (EQ-5D) effect value [21] and a patient questionnaire about the dosage form.

TEAEs, treatment-related AEs, extrapyramidal AEs, skinrelated AEs, DIEPSS, C-SSRS, PANSS, and CGI-S were assessed at the baseline and at weeks 1, 2, 4, 6, 8, 12, 16, $20,24,28,36,44$, and 52 or at the time of discontinuation of 
the blonanserin transdermal patch treatment phase in cohort 1 and cohort 2. In addition, the DIEPSS, C-SSRS, PANSS, and CGI-S assessments were also performed at baseline and at weeks 1, 2, 4, and 6 of the blonanserin tablet treatment phase in cohort 1. DAI-10 was used to obtain information relating to patient adherence. DAI-10 and EQ-5D were assessed at baseline and at weeks 6,28 , and 52 of the blonanserin transdermal patch treatment phase, and a questionnaire about the dosage form was conducted at Week 52 of the blonanserin transdermal patch treatment phase in both cohorts. EQ-5D-5L was used with Japan tariff to obtain index scores [22]. The baseline of the patch treatment phase was defined as 6 weeks after starting treatment with blonanserin tablets in cohort 1 and within 2 days of blonanserin patch application in cohort 2 .

Patient compliance with treatment was calculated from the physician-recorded patient's remainder of unused drugs and the number of prescriptions, without measuring blood blonanserin concentration.

\subsection{Statistical Analysis}

The sample size of the study was not statistically evaluated. However, assuming a drop-out rate of approximately $50 \%$, it was estimated that a sample size of 200 would be required in order to assess 100 patients for 52 weeks in accordance with the Japanese guidelines (Pharmaceutical Affairs Bureau Notification No. 592).

The Kaplan-Meier method was used for the analysis of time to treatment discontinuation. Median treatment duration and $95 \%$ confidence intervals (CIs) were calculated by cohort. Patient demographics were summarized descriptively, as were efficacy, safety, pharmacokinetic, and other endpoints by cohort and study visit. All analyses were conducted in the safety analysis set, which included patients with at least one administration of blonanserin transdermal patches during the patch treatment phase. Missing data were not imputed, except for the Week 52 (LOCF) evaluation.

For repeated measurements, summary statistics for the observed value and change from baseline value (denoted by change) are based on subjects with both a baseline and target visit observation. The last observed score at Week 52 is shown as imputed data using the LOCF method.

\section{Results}

\subsection{Patient Disposition and Baseline Characteristics}

A total of 223 patients consented to participate in this study, 117 in cohort 1 (Fig. 2a) and 106 in cohort 2
(Fig. 2b). Of the 117 patients enrolled in cohort 1, 108 were treated with blonanserin tablets, and 97 went on to receive blonanserin patches and were included in the safety analysis set. Forty patients $(41.2 \%)$ in cohort 1 discontinued treatment with blonanserin transdermal patches. The most common reason for discontinuation was AEs (18.6\%). Overall, 103 of the 106 patients who entered cohort 2 were treated with blonanserin transdermal patches and were included in the safety analysis set. Forty-four patients $(42.7 \%)$ discontinued treatment with blonanserin transdermal patches; the most common reason was withdrawal of consent $(20.4 \%)$.

In the overall safety cohort $(n=200), 91$ patients were male (45.5\%) and the mean (SD) age of patients was 43.8 (13.50) years (Table 1). Patients had been diagnosed with schizophrenia a mean (SD) of 15.4 (12.15) years before study entry. The mean (SD) baseline PANSS total score was 67.0 (22.0), and approximately two-thirds $(n=135 ; 67.5 \%)$ had more negative than positive symptoms on the baseline PANSS assessment. Patient characteristics were similar in the two cohorts (Table 1).

\subsection{Treatment Status and Continuation}

The median duration of blonanserin transdermal patch treatment was 353 days (range 2-371) in cohort 1 and 350 days (range $4-378$ ) in cohort 2 . The mean (SD) daily blonanserin transdermal patch dosage was 55.2 (17.21) mg/ day in cohort 1 and $56.0(15.91) \mathrm{mg} /$ day in cohort 2. At 28 weeks, $64.9 \%$ of patients (95\% CI 54.6-73.5) in cohort 1 and $66.0 \%$ (95\% CI 56.0-74.3) in cohort 2 were still using blonanserin transdermal patches. At 52 weeks, the treatment retention rate was 58.8\% (95\% CI 48.3-67.8) in cohort 1 and 57.3\% (95\% CI 47.2-66.2) in cohort 2 (Fig. $\mathrm{S} 1$; see the ESM).

Compliance with medication was $98.6 \%$ and $98.4 \%$ in cohort 1 and 2 , respectively.

In terms of concomitant medications used in cohort 1 and cohort 2, 15.5\% (15/97) and 21.4\% (22/103) of patients used antipsychotics, 33.0\% (32/97) and 22.3\% (23/103) used antiparkinsonian drugs, $40.2 \%$ (39/97) and 40.8\% (42/103) used psychotropics, and 69.1\% (68/103) and 66.0\% (67/97) used sleeping pills during treatment, respectively.

\subsection{Safety}

AEs were observed in 174 patients $(87.0 \%)$, with a similar incidence in cohort $1(84.5 \%)$ and cohort $2(89.3 \%)$ (Table 2). Most AEs were mild or moderate in severity; seven patients (3.5\%) developed severe AEs. A total of 


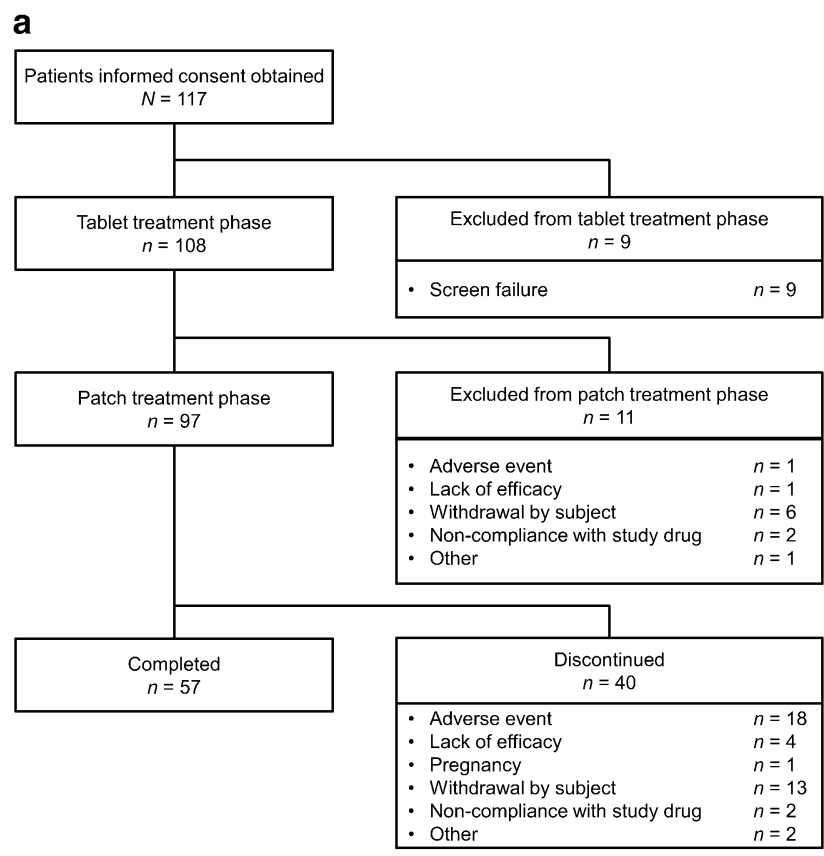

\section{b}

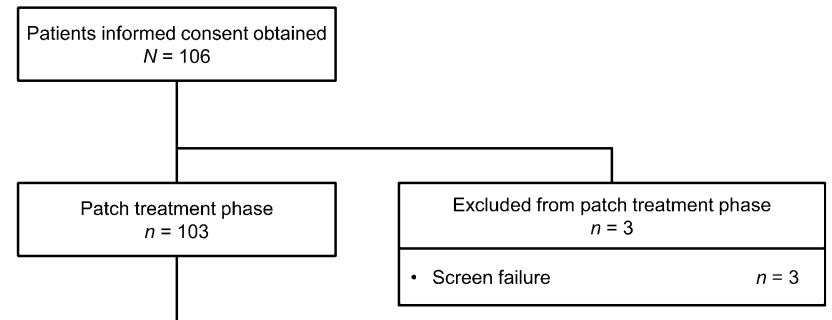

Fig. 2 Patient flow for $\mathbf{a}$ cohort 1 and $\mathbf{b}$ cohort 2

Table 1 Patient background and clinical characteristics at study entry (prior to any blonanserin therapy)

\begin{tabular}{|c|c|c|c|}
\hline & $\begin{array}{l}\text { Cohort } 1 \\
n=97\end{array}$ & $\begin{array}{l}\text { Cohort } 2 \\
n=103\end{array}$ & $\begin{array}{l}\text { Overall } \\
N=200\end{array}$ \\
\hline Male sex, $n(\%)$ & $43(44.3)$ & 48 (46.6) & $91(45.5)$ \\
\hline Mean (SD) age (years) & $44.1(14.43)$ & $43.6(12.63)$ & $43.8(13.50)$ \\
\hline Mean (SD) weight (kg) & $65.22(17.637)$ & $66.28(15.145)$ & $65.77(16.368)$ \\
\hline Mean (SD) BMI (kg/m²) & $24.68(5.429)$ & $24.70(4.857)$ & $24.69(5.129)$ \\
\hline \multicolumn{4}{|l|}{ Hospitalization status at baseline, $n(\%)$} \\
\hline Inpatient & $8(8.2)$ & $17(16.5)$ & $25(12.5)$ \\
\hline Outpatient & $89(91.8)$ & $86(83.5)$ & $175(87.5)$ \\
\hline Patients with concomitant diseases, $n(\%)$ & $87(89.7)$ & $79(76.7)$ & $166(83.0)$ \\
\hline Mean (SD) age at schizophrenia onset, years & $29.1(12.60)$ & $27.8(9.55)$ & $28.4(11.15)$ \\
\hline Mean (SD) total schizophrenia episodes & $1.9(1.43)$ & $1.8(1.32)$ & $1.8(1.37)$ \\
\hline Mean (SD) duration of illness ${ }^{\mathrm{a}}$, years & $14.9(12.72)$ & $15.8(11.63)$ & $15.4(12.15)$ \\
\hline Mean (SD) duration of current episode ${ }^{\mathrm{b}}$, months & $104.7(108.87)$ & $104.1(93.38)$ & $104.4(100.89)$ \\
\hline Mean (SD) PANSS total score & $66.4(22.73)$ & $67.5(21.34)$ & $67.0(21.98)$ \\
\hline Positive > negative subscale score, $n(\%)$ & $24(24.7)$ & $22(21.4)$ & $46(23.0)$ \\
\hline Positive $=$ negative subscale score, $n(\%)$ & $8(8.2)$ & $11(10.7)$ & $19(9.5)$ \\
\hline Positive $<$ negative subscale score, $n(\%)$ & $65(67.0)$ & $70(68.0)$ & $135(67.5)$ \\
\hline Mean (SD) CGI-S score & $3.5(1.07)$ & $3.4(1.11)$ & $3.4(1.09)$ \\
\hline Mean (SD) DIEPSS total score ${ }^{c}$ & $0.6(1.55)$ & $0.8(1.56)$ & $0.7(1.55)$ \\
\hline
\end{tabular}

$B M I$ body mass index, CGI-S Clinical Global Impressions - Severity of Illness Scale, DIEPSS Drug-Induced Extrapyramidal Symptoms Scale, PANSS Positive and Negative Syndrome Scale, $S D$ standard deviation

${ }^{a}$ Time from the initial episode of schizophrenia to provision of informed consent

${ }^{\mathrm{b}}$ Time from the onset of the current episode to provision of informed consent

${ }^{c}$ Excludes overall severity 
13 serious AEs occurred in 12 patients (6.0\%), of which six occurred in cohort 1 and six occurred in cohort 2 . Serious AEs were schizophrenia $(n=6)$ and seven AEs $(n=6)$, which included impulse-control disorder, fracture, epistaxis, asthma, pneumonia aspiration, pneumonia hemophilus, and pneumonia. No AEs were fatal. Discontinuation resulted from AEs in $18.6 \%$ (cohort 1) and $11.7 \%$ (cohort 2) of patients and from withdrawal of consent in $13.4 \%$ (cohort 1) and $20.4 \%$ (cohort 2) of patients. The most common AEs included nasopharyngitis $(n=62$, $31.0 \%)$, application site erythema $(n=45,22.5 \%)$, application site pruritus $(n=23,11.5 \%)$, and akathisia $(n=20$, $10.0 \%$ ) (Table 3).

A total of 78 extrapyramidal AEs occurred in 51 patients $(25.5 \%)$ in the overall patient population. The most frequent extrapyramidal AE was akathisia $(n=20$, $10.0 \%)$. None of the extrapyramidal AEs were serious. Among those who were not using antiparkinsonian drugs at study entry, $15.6 \%(12 / 77)$ in cohort 1 and $20.0 \%$ $(20 / 100)$ in cohort 2 initiated antiparkinsonian drugs during the treatment period.

Skin-related TEAEs at the site of patch application were observed in $40.2 \%$ and $42.7 \%$ in cohort 1 and 2 , respectively. Of these, 116 skin-related AEs in 78 patients (39.0\%) were considered to be treatment-related. Skinrelated AEs were mostly mild, and none were serious. Thirty-three percent of skin-related TEAEs occurred in week 1-13 and 7.9\% in week 14-26. Skin-related TEAEs reported in $\geq 5 \%$ of patients included application site erythema, pruritus, and dermatitis. Approximately $22 \%$ of skin-related AEs required treatment. Topical steroids and topical antihistamines were used by $7.2 \%$ and $13.6 \%$ of patients in cohorts 1 and 2, respectively. Among the patients who showed skin-related TEAEs $(n=83)$, approximately half $(n=44)$ received any treatment, including topical steroids $(n=21)$, topical antihistamines $(n=7)$, and others ( $n=16$; e.g., moisturizer). Most patients with skin-related TEAEs recovered by the end of the trial. Seven patients discontinued the study due to skin-related TEAEs.

None of the patients in the safety analysis set developed bullae during the blonanserin transdermal patch treatment phase according to the skin irritation assessment scores. The maximum scores were vesicle $(+++)$ in two patients $(1.0 \%)$, erythema/edema $(++)$ in $12(6.0 \%)$, erythema $(+)$ in $48(24.0 \%)$, and mild erythema $( \pm)$ in 73 patients $(36.5 \%)$.

There were no clinically significant changes in laboratory tests or examinations, including prolactin level, body weight, metabolism-related parameters, and QTc interval. The mean (SD) change in body weight was -0.04 (4.561) $\mathrm{kg}$ and $-0.67(6.841) \mathrm{kg}$ in cohort 1 and cohort 2 , respectively.

The mean (SD) change from baseline in total DIEPSS score at Week 52 (LOCF) was - 0.1 (1.55), indicating no marked effect on EPS in patients who completed this assessment $(n=117)$.

In total, 71 patients had a prior history of suicidal ideation and were excluded from the C-SSRS assessment. Of the patients who answered "No" to all C-SSRS categories at baseline $(n=129), 13$ patients $(10.1 \%)$ were evaluated as having emergence of suicidal ideation. Among patients who answered "No" to all C-SSRS suicidal behavior categories at baseline $(n=172)$, one $(0.6 \%)$ was evaluated as having suicidal behavior during blonanserin transdermal patch treatment. No completed suicide was reported.

\subsection{Efficacy}

\subsubsection{Cohort 1}

In cohort 1 , the PANSS total score decreased by a mean (SD) of 2.9 (7.2) points from the start of the tablet treatment until the last assessment before the switch to patches, and did not increase after switching treatment

Table 2 Summary of TEAEs

\begin{tabular}{|c|c|c|c|c|c|c|}
\hline & \multicolumn{2}{|l|}{$\begin{array}{l}\text { Cohort } 1 \\
n=97\end{array}$} & \multicolumn{2}{|l|}{$\begin{array}{l}\text { Cohort } 2 \\
n=103\end{array}$} & \multicolumn{2}{|l|}{$\begin{array}{l}\text { Overall } \\
N=200\end{array}$} \\
\hline & $n(\%)$ & Events & $n(\%)$ & Events & $n(\%)$ & Events \\
\hline TEAE & $82(84.5)$ & 306 & $92(89.3)$ & 442 & $174(87.0)$ & 748 \\
\hline Leading to death & 0 & 0 & 0 & 0 & 0 & 0 \\
\hline Serious & $6(6.2)$ & 6 & $6(5.8)$ & 7 & $12(6.0)$ & 13 \\
\hline $\begin{array}{l}\text { Leading to treatment dis- } \\
\text { continuation }\end{array}$ & $16(16.5)$ & 19 & $12(11.7)$ & 15 & $28(14.0)$ & 34 \\
\hline Severe & $4(4.1)$ & 4 & $3(2.9)$ & 3 & $7(3.5)$ & 7 \\
\hline Extrapyramidal & $21(21.6)$ & 27 & $30(29.1)$ & 51 & $51(25.5)$ & 78 \\
\hline Skin-related & $39(40.2)$ & 51 & $44(42.7)$ & 72 & $83(41.5)$ & 123 \\
\hline
\end{tabular}

TEAE treatment-emergent adverse event 
Table 3 TEAEs that were reported in $\geq 2 \%$ of patients

\begin{tabular}{|c|c|c|c|}
\hline & \multicolumn{3}{|c|}{ TEAE, $n(\%)$} \\
\hline & $\begin{array}{l}\text { Cohort } 1 \\
n=97\end{array}$ & $\begin{array}{l}\text { Cohort } 2 \\
n=103\end{array}$ & $\begin{array}{l}\text { Overall } \\
N=200\end{array}$ \\
\hline Subjects with any TEAE & $82(84.5)$ & $92(89.3)$ & $174(87.0)$ \\
\hline Endocrine disorders & $7(7.2)$ & $7(6.8)$ & $14(7.0)$ \\
\hline Hyperprolactinemia & $7(7.2)$ & $7(6.8)$ & $14(7.0)$ \\
\hline Gastrointestinal disorders & $23(23.7)$ & $34(33.0)$ & $57(28.5)$ \\
\hline Dental caries & $5(5.2)$ & $8(7.8)$ & $13(6.5)$ \\
\hline Diarrhea & $5(5.2)$ & $5(4.9)$ & $10(5.0)$ \\
\hline Vomiting & $7(7.2)$ & $3(2.9)$ & $10(5.0)$ \\
\hline Constipation & $2(2.1)$ & $5(4.9)$ & $7(3.5)$ \\
\hline Nausea & $3(3.1)$ & $3(2.9)$ & $6(3.0)$ \\
\hline Salivary hypersecretion & $3(3.1)$ & $3(2.9)$ & $6(3.0)$ \\
\hline Stomatitis & $0(0.0)$ & $4(3.9)$ & $4(2.0)$ \\
\hline General disorders and administration site conditions & $42(43.3)$ & $49(47.6)$ & $91(45.5)$ \\
\hline Application site erythema & $18(18.6)$ & $27(26.2)$ & $45(22.5)$ \\
\hline Application site pruritus & $9(9.3)$ & $14(13.6)$ & $23(11.5)$ \\
\hline Application site dermatitis & $8(8.2)$ & $2(1.9)$ & $10(5.0)$ \\
\hline Application site discoloration & $4(4.1)$ & $2(1.9)$ & $6(3.0)$ \\
\hline Malaise & $1(1.0)$ & $3(2.9)$ & $4(2.0)$ \\
\hline Infections and infestations & $32(33.0)$ & $41(39.8)$ & $73(36.5)$ \\
\hline Nasopharyngitis & $29(29.9)$ & $33(32.0)$ & $62(31.0)$ \\
\hline Pharyngitis & $2(2.1)$ & $2(1.9)$ & $4(2.0)$ \\
\hline Injury, poisoning and procedural complications & $12(12.4)$ & $13(12.6)$ & $25(12.5)$ \\
\hline Contusion & $3(3.1)$ & $3(2.9)$ & $6(3.0)$ \\
\hline Excoriation & $3(3.1)$ & $2(1.9)$ & $5(2.5)$ \\
\hline Ligament sprain & $1(1.0)$ & $3(2.9)$ & $4(2.0)$ \\
\hline Investigations & $10(10.3)$ & $28(27.2)$ & $38(19.0)$ \\
\hline Blood prolactin increased & $2(2.1)$ & $12(11.7)$ & $14(7.0)$ \\
\hline Weight increased & $3(3.1)$ & $7(6.8)$ & $10(5.0)$ \\
\hline Blood CPK increased & $1(1.0)$ & $3(2.9)$ & $4(2.0)$ \\
\hline Musculoskeletal and connective tissue disorders & $15(15.5)$ & $15(14.6)$ & $30(15.0)$ \\
\hline Back pain & $8(8.2)$ & $5(4.9)$ & $13(6.5)$ \\
\hline Arthralgia & $1(1.0)$ & $3(2.9)$ & $4(2.0)$ \\
\hline Muscle rigidity & $1(1.0)$ & $3(2.9)$ & $4(2.0)$ \\
\hline Nervous system disorders & $26(26.8)$ & $35(34.0)$ & $61(30.5)$ \\
\hline Akathisia & $6(6.2)$ & $14(13.6)$ & $20(10.0)$ \\
\hline Tremor & $4(4.1)$ & $10(9.7)$ & $14(7.0)$ \\
\hline Headache & $6(6.2)$ & $1(1.0)$ & $7(3.5)$ \\
\hline Dizziness & $3(3.1)$ & $3(2.9)$ & $6(3.0)$ \\
\hline Dyskinesia & $2(2.1)$ & $4(3.9)$ & $6(3.0)$ \\
\hline Dystonia & $4(4.1)$ & $2(1.9)$ & $6(3.0)$ \\
\hline Bradykinesia & $2(2.1)$ & $3(2.9)$ & $5(2.5)$ \\
\hline Somnolence & $1(1.0)$ & $4(3.9)$ & $5(2.5)$ \\
\hline Psychiatric disorders & $25(25.8)$ & $28(27.2)$ & $53(26.5)$ \\
\hline Insomnia & $7(7.2)$ & $11(10.7)$ & $18(9.0)$ \\
\hline Schizophrenia & $9(9.3)$ & $9(8.7)$ & $18(9.0)$ \\
\hline Restlessness & $4(4.1)$ & $2(1.9)$ & $6(3.0)$ \\
\hline Skin and subcutaneous tissue disorders & $7(7.2)$ & $15(14.6)$ & $22(11.0)$ \\
\hline Eczema & $3(3.1)$ & $2(1.9)$ & $5(2.5)$ \\
\hline
\end{tabular}

$C P K$ creatine phosphokinase, TEAE treatment-emergent adverse event 
from blonanserin tablets to patches (Fig. 3a). The mean (SD) change from baseline in PANSS total score at Week 52 and Week 52 (LOCF) was - 3.5 (8.4) and -0.1 (11.6), respectively. The PANSS subscale scores (Figs. S2-S4, see the ESM) and PANSS 5-factor scores (Figs. S5-S9) did not increase during the 52 weeks of the blonanserin transdermal patch treatment phase. The PANSS scores did not change after switching from tablets to patches.

The CGI-S score decreased by a mean (SD) of $0.1(0.41)$ from the start of the tablet treatment until the last assessment before the switch to patches, and the mean (SD) change from baseline in CGI-S score at Week 52 and Week 52 (LOCF) was $-0.1(0.65)$ and $0(0.76)$, respectively. The CGI-S score did not increase after switching treatment from blonanserin tablets to patches during 52 weeks of treatment (Fig. S10).

\subsubsection{Cohort 2}

In cohort 2, the PANSS total score decreased over the 52 weeks of treatment with the blonanserin transdermal patch (Fig. 3b). The mean (SD) change from baseline in PANSS total score at Week 52 and Week 52 (LOCF) was -9.2 (15.1) and - 3.4 (15.3), respectively. The mean (SD) changes in the PANSS positive scores, negative scores, and general psychopathology subscale scores at Week 52 (LOCF) were -0.7 (4.55), -1.6 (4.35), and -1.1 (8.05), respectively. PANSS subscale scores (Figs. S2-S4) and 5-factor scores (Figs. S5-S9) showed small decreases over the first 6-8 weeks of treatment and then remained stable over the remainder of the assessment period. The PANSS total, subscale scores, and 5-factor scores showed gradual decrease throughout the treatment period.

The CGI-S score did not change throughout the blonanserin transdermal patch treatment (Fig. S10). The mean (SD)
Fig. 3 Mean (SD) PANSS total scores at each timepoint in a cohort 1 and $\mathbf{b}$ cohort 2 . The PANSS score at each timepoint was shown as observed case using pairwise deletion analyses. PANSS Positive and Negative Syndrome Scale, $S D$ standard deviation
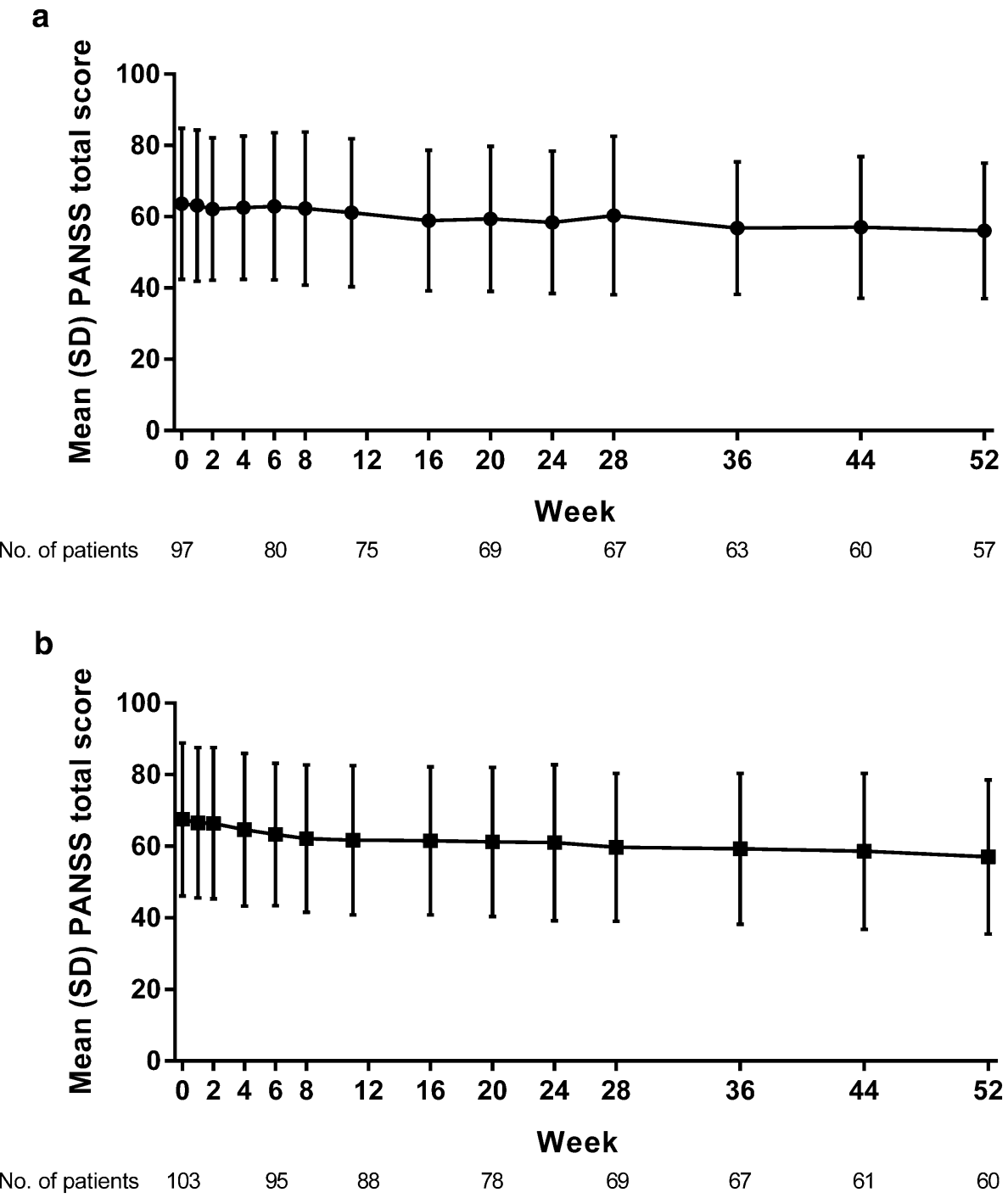
change from baseline in CGI-S score at Week 52 and Week 52 (LOCF) was $-0.6(0.83)$ and $-0.2(1.03)$, respectively.

\subsection{Patient-Reported Outcomes}

In patients who completed 52 weeks of blonanserin transdermal patch treatment $(n=129)$, the mean (SD) change in EQ-5D score from baseline to 52 weeks was -0.0172 $(0.16968)$ for both cohorts combined. In the intention-totreat population of the combined cohorts $(n=200)$, the mean (SD) change from baseline in EQ-5D score at the last assessment (either Week 52 or the last on-treatment assessment) was -0.0365 (0.17603).

After 52 weeks of blonanserin transdermal patch treatment, the total DAI-10 score increased or remained unchanged compared with baseline in 82 of the 129 patients $(63.6 \%)$ for whom these data were available. As a positive DAI-10 score is associated with improved adherence [23], we set the minimum positive score value of 2 as the cut-off score between positive and negative attitude toward treatment, because 2 is the smallest positive number. DAI-10 scores have been maintained during the study (Table 4); however, in 165 patients who had a DAI-10 score $\geq 2$ at baseline, $30(18.2 \%)$ reverted to a score of $<2$ at Week 52 (LOCF), whereas $48.6 \%$ (17/35) of patients with a score of $<2$ at baseline reported a score $\geq 2$ at Week 52 (LOCF). In the patient questionnaire about dosage form, most patients indicated that it would be better if patch formulations of any treatment were available ( $n=135,69.6 \%$; Table 5). Approximately onethird of patients indicated that, compared with taking tablets, continuing treatment with patches would be easier $(n=62$, $32.0 \%)$ or at least as easy $(n=69,35.6 \%)$. Forty-one percent of patients indicated that they would use blonanserin transdermal patches in the future $(n=79,40.7 \%)$.

\section{Discussion}

This was the first study to evaluate the long-term safety and efficacy of blonanserin transdermal patches in Japanese patients with schizophrenia and showed that blonanserin

Table 4 Number of patients with total DAI- 10 score $<2$ or $\geq 2$ before and after the blonanserin transdermal patch treatment phase

\begin{tabular}{lll}
\hline & \multicolumn{2}{l}{ Number of patients $(\%)$} \\
\cline { 2 - 3 } & DAI-10 score $<2$ & DAI-10 score $\geq 2$ \\
\hline $\begin{array}{l}\text { Before the treatment } \\
\text { baseline }\end{array}$ & $35(17.5)$ & $165(82.5)$ \\
Week 52 (LOCF) & $48(24.0)$ & $152(76.0)$ \\
\hline
\end{tabular}

DAI-10 Drug Attitude Inventory-10, LOCF last observation carried forward transdermal patches are well tolerated and maintain efficacy in the long-term treatment of schizophrenia.

As expected, the long-term safety profile of blonanserin patches was similar to, or better than, that of blonanserin tablets. The incidence of treatment-related AEs observed in the present study (68.5\%) was similar to that reported in the previous long-term studies of blonanserin tablets (72.1\% and $68.5 \%)[5,7]$. The incidence of extrapyramidal AEs, such as akathisia and tremor, was lower in the present study ( $10.0 \%$ and $7.0 \%$, respectively) than in the long-term studies of blonanserin tablets (akathisia $17.1 \%-32.8 \%$, tremor $15.9 \%-21.3 \%)[5,7]$. This lower incidence of overall AEs or

Table 5 Dosage form questionnaire

\begin{tabular}{|c|c|c|c|c|}
\hline & & $\begin{array}{l}\text { Cohort } 1 \\
n=97\end{array}$ & $\begin{array}{l}\text { Cohort } 2 \\
n=103\end{array}$ & $\begin{array}{l}\text { Overall } \\
N=200\end{array}$ \\
\hline \multicolumn{5}{|c|}{ Question 1. Would you like the patch dosage form to be available? } \\
\hline \multirow[t]{3}{*}{ Completed } & $n$ & 57 & 59 & 116 \\
\hline & Yes & $42(73.7)$ & $52(88.1)$ & $94(81.0)$ \\
\hline & No & $15(26.3)$ & 7 (11.9) & $22(19.0)$ \\
\hline \multirow[t]{3}{*}{ Discontinued } & $n$ & 39 & 39 & 78 \\
\hline & Yes & $22(56.4)$ & $19(48.7)$ & $41(52.6)$ \\
\hline & No & $17(43.6)$ & $20(51.3)$ & $37(47.4)$ \\
\hline \multirow[t]{3}{*}{ Overall } & $n$ & 96 & 98 & 194 \\
\hline & Yes & $64(66.7)$ & $71(72.4)$ & 135 (69.6) \\
\hline & No & $32(33.3)$ & $27(27.6)$ & $59(30.4)$ \\
\hline
\end{tabular}

Question 2. Is it easy for you to continue treatment with patches compared with tablets?

$\begin{array}{llccr}\text { Completed } & n & 57 & 59 & 116 \\ & \text { Yes } & 19(33.3) & 27(45.8) & 46(39.7) \\ & \text { Equal } & 24(42.1) & 21(35.6) & 45(38.8) \\ \text { Discontinued } & \text { No } & 14(24.6) & 11(18.6) & 25(21.6) \\ & n & 39 & 39 & 78 \\ & \text { Yes } & 10(25.6) & 6(15.4) & 16(20.5) \\ & \text { Equal } & 9(23.1) & 15(38.5) & 24(30.8) \\ \text { Overall } & \text { No } & 20(51.3) & 18(46.2) & 38(48.7) \\ & n & 96 & 98 & 194 \\ & \text { Yes } & 29(30.2) & 33(33.7) & 62(32.0) \\ & \text { Equal } & 33(34.4) & 36(36.7) & 69(35.6) \\ & \text { No } & 34(35.4) & 29(29.6) & 63(32.5)\end{array}$

Question 3. Would you like to use the patches that you used during this study in the future?

\begin{tabular}{llccc} 
Completed & $n$ & 57 & 59 & 116 \\
& Yes & $25(43.9)$ & $36(61.0)$ & $61(52.6)$ \\
& No & $32(56.1)$ & $23(39.0)$ & $55(47.4)$ \\
Discontinued & $n$ & 39 & 39 & 78 \\
& Yes & $10(25.6)$ & $8(20.5)$ & $18(23.1)$ \\
\multirow{5}{*}{ Overall } & No & $29(74.4)$ & $31(79.5)$ & $60(76.9)$ \\
& $n$ & 96 & 98 & 194 \\
& Yes & $35(36.5)$ & $44(44.9)$ & $79(40.7)$ \\
& No & $61(63.5)$ & $54(55.1)$ & $115(59.3)$ \\
\hline
\end{tabular}


extrapyramidal TEAEs could possibly be due to a less rapid increase and stable transition in plasma blonanserin levels with the transdermal patches compared with the oral formulation. When plasma drug concentrations antagonizing dopamine $\mathrm{D}_{2}$ receptors are maintained stably, maximum blood concentration $\left(C_{\max }\right)$-dependent AEs could be suppressed. Hyperprolactinemia was observed in $7.0 \%$ of patients in the present study, which was less than that previously observed with blonanserin tablets (34.4\% and 29.6\%) [5, 7]. Weight gain was observed in $5.0 \%$ of patients in this study and $8.2 \%$ and $7.2 \%$ in the previous studies with blonanserin tablets $[5,7]$.

In long-term studies conducted in patients with schizophrenia treated with other antipsychotics approved in Japan, the overall incidence of AEs at 48 or 52 weeks was between 84 and 97\% [24-27], and our study showed similar safety findings (87\%). The overall incidence of extrapyramidal AEs was $25.5 \%$ in this study, which was lower than that found with blonanserin tablets (35.8\%) [7] and lower than (32.9\%) [24] or similar to (22.2\%) [25] that of other antipsychotics approved in Japan. The proportion of patients who were administered antiparkinsonian drugs was 18\% (32/177) in this study, which was lower than that reported in the previous studies with other antipsychotics approved in Japan $(93 \%, 25 \%$, and $81 \%)$ [7, 24, 25].

Skin-related AEs are characteristic of patch formulations. In the present study, skin-related AEs were observed in 41.5\% of patients, which was higher than that reported in the shortterm phase III, placebo-controlled study of blonanserin transdermal patches (13.3\%-18.6\%) [13]. In this study, the substrate and the drug substance were not distinguished during the collection of AEs. It should be noted, however, that in the short-term phase III study, the incidence of AEs at the application site was higher in the active patch group than in the placebo patch group. Although the number of cases increased due to the long duration of treatment, all skin-related AEs observed in the present study were mild or moderate in severity. They tended to occur early in treatment and were appropriately managed with topical therapies. Two patients discontinued the study because of skin-related AEs; however, the proportion of patients who used treatment for skin-related AEs was similar to that of the phase III study [13].

The rate of treatment discontinuation observed with blonanserin transdermal patches in the present study was similar to rates reported in long-term studies of other antipsychotic drugs conducted in Japan [24-27]. No marked increase in treatment discontinuation was observed after switching from tablets to patches in cohort 1 . In the phase III study, patients in the 80-mg treatment group switched from their prior treatment without tapering, and no special AEs that were possibly due to switching were observed [13]. Taken together, these data suggest that the blonanserin patches are well tolerated when switching from blonanserin tablets or from other antipsychotic drugs, although further study is warranted.

Suicidal ideation is an important parameter to assess in the treatment of patients with schizophrenia. However, it was difficult to compare the results of the present study with those of other studies, because none of them assessed suicidal ideation or behavior using the C-SSRS [24-27]. In the phase III study of blonanserin transdermal patches, the incidence of suicidal ideation evaluated using the C-SSRS was $5.6 \%$ with $40 \mathrm{mg}$ and $3.1 \%$ with $80 \mathrm{mg}$ blonanserin transdermal patches [13], which was lower than the incidence observed in the present study (10.1\%). Further research, such as a study in the real-world clinical setting, is warranted to evaluate the influence of blonanserin transdermal patch therapy on suicidal thoughts and behavior.

The PANSS and CGI-S scores remained relatively stable throughout the study, and no apparent increases were noted. In addition, both the positive and negative symptoms of schizophrenia were stabilized with blonanserin transdermal patches. Continuous control of symptoms is one of the main goals of long-term pharmacotherapy in schizophrenia. Considering the fact that relapse or worsening of symptoms during treatment is one of the risk factors for non-adherence to treatment [28], sustained efficacy over an extended period could help to maintain a patient's long-term engagement with treatment. In addition, many of the patients enrolled in this study were considered to have chronic schizophrenia based on the mean duration of disease and of the current episode, and the study included a high proportion of patients with higher negative than positive PANSS symptom scores [29]. Over $98 \%$ of patients had been treated with antipsychotics, which are generally more effective in reducing positive symptoms than negative symptoms [30]. These factors could have influenced the effectiveness of blonanserin transdermal patches in the present study. The 6-week, randomized, phase III study of blonanserin transdermal patches showed efficacy in patients with acute phase schizophrenia. In this long-term study, similar efficacy was shown in patients with chronic schizophrenia. Taken together, it is expected that blonanserin transdermal patch therapy may be effective for patients in either acute or chronic phases of schizophrenia.

Continuous drug therapy is important to control schizophrenia, particularly in the maintenance/stable phase. Although poor treatment adherence increases the risk of rehospitalization, patients with schizophrenia often stop taking their medication [31]. While there are a number of reasons for treatment discontinuation, one key factor is patients' low willingness to participate in the treatment process [32]. Shared decision making (SDM) that includes patients and medical staff, as well as physicians, is a method of improving treatment adherence, for example, by presenting patients with a choice of treatment options or deciding on a specific treatment based on the patient's intentions or preferences. 
SDM decreases the frequency of re-hospitalizations in patients with schizophrenia [33]. To support SDM, it is important to include patient-reported outcomes and measures of patients' attitude to treatment, such as DAI-10 scores [34], in the evaluation of treatment [11]. In particular, positive DAI-10 scores are associated with improved treatment adherence [23].

Based on the DAI-10 scores, patients' attitudes towards blonanserin transdermal patches were generally positive. Most patients with a DAI-10 score $\geq 2$ at baseline had the same DAI-10 score after 52 weeks of treatment, with $18.2 \%$ reverting to a score of $<2$ at Week 52 (LOCF). Among patients with a score of $<2$ at baseline, $48.6 \%$ reported a score $\geq 2$ at Week 52 (LOCF). In addition, no marked changes in the EQ-5D scores were observed, indicating maintenance of stable quality of life.

In the patient questionnaire asking specifically about blonanserin transdermal patch treatment, $60 \%$ of patients indicated that they would not use blonanserin transdermal patches in the future, but this included patients who had discontinued treatment. In patients who completed the study, more than half (52.6\%) said they would like to use the patches. In addition, the majority of patients $(69.6 \%)$ would prefer to have transdermal patches available as a potential treatment option.

Taken together, these DAI-10 scores and results from a questionnaire indicate that the positive attitude toward blonanserin transdermal patch treatment held by a certain proportion of patients did not change during long-term treatment. As the patient's positive attitude is critical for improving treatment adherence, the use of the patch formulation of blonanserin may help patients to achieve a positive treatment outcome.

Limitations of this study include its open-label, uncontrolled design and the absence of statistical evaluation of efficacy endpoints. As this is an open-label study without an active or placebo comparator, it is unclear if the same cohort would have improved similarly with an alternate treatment regimen or no treatment at all. In addition, the number of patients who had been previously treated with blonanserin was higher than in previous clinical studies, suggesting that recruitment may have been biased. Further, it is difficult to determine whether positive results shown in this study were related to improved adherence. Although the patients in this study showed good compliance to treatment, adherence should be evaluated according to patient's attitude toward the treatment. For supporting information, we used DAI-10 score and an original questionnaire; however, additional data to analyze patient's adherence is needed. In addition, it is also unclear whether improvement was related to longer time on an antipsychotic medication or other characteristics of the medication or formulation. Further studies with a controlled design may help answer these questions.

\section{Conclusion}

This study demonstrates the safety and efficacy of blonanserin transdermal patches in the long-term treatment of patients with schizophrenia, including after switching from blonanserin tablets. The long-term safety profile of blonanserin transdermal patches was similar to, or better than, that of blonanserin tablets. The majority of skin-related AEs, which were mild or moderate in severity, occurred early in treatment and were appropriately managed with topical therapies. Efficacy measures did not worsen during treatment with blonanserin transdermal patches. In addition, patients' attitudes to blonanserin transdermal patch therapy were generally positive, and these positive attitudes did not decrease during long-term treatment.

Acknowledgements The authors would like to thank the participants of this study and all investigators and staff involved in conducting the study. The authors also thank Yoshiko Okamoto and Georgii Filatov of inScience Communications, Springer Healthcare, for medical writing support in the preparation of this manuscript, which included drafting and redrafting of material in response to author contributions. Funding for medical writing assistance was provided by Sumitomo Dainippon Pharma Co., Ltd.

Data Availability The data that support the findings of this study are available from the corresponding author, Hiroshi Nakamura, upon reasonable request.

\section{Compliance with Ethical Standards}

Ethical approval The study protocol was approved by the institutional review boards of all participating institutions. This study was also conducted in compliance with the ethical principles stated in the Declaration of Helsinki and with the Good Clinical Practice (GCP) guidelines and other relevant Japanese regulations.

Informed consent Written informed consent was obtained from all patients or legal guardians (if the patient was $<20$ years old or was hospitalized for medical care and protection) prior to the study based on institutional guidelines.

Funding This study was funded by Sumitomo Dainippon Pharma Co., Ltd. The open access fee was also paid by Sumitomo Dainippon Pharma Co., Ltd.

Conflict of interest NI reports personal fees from Otsuka, Sumitomo Dainippon, Janssen, Eli-Lilly, and Pfizer and grants from Otsuka and Daiichi-Sankyo outside the submitted work. JI reports grants from Sumitomo Dainippon during the conduct of the study and personal fees from Meiji Seika Pharma, MSD, Astellas, Novartis, Pfizer, Otsuka, Eli Lilly, Takeda, and Eisai outside the submitted work. TH reports personal fees from Meiji Seika Pharma, MSD, Allergan, Eisai, Pfizer, Janssen, Lundbeck, Shionogi, Yoshitomi, Kyowa Hakko Kirin, Mo- 
chida, Otsuka, Sumitomo Dainippon, Mitsubishi Tanabe, Eli Lilly, and Takeda outside the submitted work. IN, MM, YK, and HN are employees of Sumitomo Dainippon Pharma Co., Ltd.

Open Access This article is licensed under a Creative Commons Attribution-NonCommercial 4.0 International License, which permits any non-commercial use, sharing, adaptation, distribution and reproduction in any medium or format, as long as you give appropriate credit to the original author(s) and the source, provide a link to the Creative Commons licence, and indicate if changes were made. The images or other third party material in this article are included in the article's Creative Commons licence, unless indicated otherwise in a credit line to the material. If material is not included in the article's Creative Commons licence and your intended use is not permitted by statutory regulation or exceeds the permitted use, you will need to obtain permission directly from the copyright holder. To view a copy of this licence, visit http://creativecommons.org/licenses/by-nc/4.0/.

\section{References}

1. Deeks ED, Keating GM. Blonanserin: a review of its use in the management of schizophrenia. CNS Drugs. 2010;24(1):65-84.

2. Li H, Yao C, Shi J, Yang F, Qi S, Wang L, et al. Comparative study of the efficacy and safety between blonanserin and risperidone for the treatment of schizophrenia in Chinese patients: a doubleblind, parallel-group multicenter randomized trial. J Psychiatr Res. 2015;69:102-9.

3. Sumitomo Dainippon Pharma Co. Ltd. Lonasen tape $20 \mathrm{mg}$, $30 \mathrm{mg}$, and $40 \mathrm{mg}$ [Japanese prescribing information]. 2019. http://www.info.pmda.go.jp/go/pack/11797A0S1025_1_03/. Accessed August 26, 2019.

4. Murasaki M. Clinical evaluation of blonanserin for schizophrenia: a randomized controlled study comparing blonanserin with haloperidol. Jpn J Clin Psychopharmacol. 2007;10(11):2059-79.

5. Murasaki M. Long-term clinical study of blonanserin for schizophrenia: a multicenter open study to determine safety and effectiveness in schizophrenic patients (Kanagawa Region Clinical Psychopharmacology Study Group). Jpn J Clin Psychopharmacol. 2007;10(12):2241-57.

6. Miura S. Clinical evaluation of blonanserin for schizophrenia: a randomized controlled study comparing blonanserin with risperidone. Jpn J Clin Psychopharmacol. 2008;11(2):297-314.

7. Kinoshita T. Long-term clinical study of blonanserin for schizophrenia: a multicenter open study to determine safety and effectiveness in schizophrenic patients (Japan-wide study). Jpn J Clin Psychopharmacol. 2008;11(1):135-53.

8. Garcia E, Robert M, Peris F, Nakamura H, Sato N, Terazawa Y. The efficacy and safety of blonanserin compared with haloperidol in acute-phase schizophrenia: a randomized, doubleblind, placebo-controlled, multicentre study. CNS Drugs. 2009;23(7):615-25.

9. Yang J, Bahk WM, Cho HS, Jeon YW, Jon DI, Jung HY, et al. Efficacy and tolerability of Blonanserin in the patients with schizophrenia: a randomized, double-blind, risperidone-compared trial. Clin Neuropharmacol. 2010;33(4):169-75.

10. Kishi T, Matsui Y, Matsuda Y, Katsuki A, Hori H, Yanagimoto $\mathrm{H}$, et al. Efficacy, tolerability, and safety of blonanserin in schizophrenia: an updated and extended systematic review and meta-analysis of randomized controlled trials. Pharmacopsychiatry. 2018;52(2):52-62.

11. De Las Cuevas C, Penate W. Explaining pharmacophobia and pharmacophilia in psychiatric patients: relationship with treatment adherence. Hum Psychopharmacol. 2015;30(5):377-83.
12. Ingersoll KS, Cohen J. The impact of medication regimen factors on adherence to chronic treatment: a review of literature. J Behav Med. 2008;31(3):213-24.

13. Iwata N, Ishigooka J, Kim W-H, Yoon B-H, Lin S-K, Sulaiman $\mathrm{AH}$, et al. Efficacy and safety of blonanserin transdermal patch in patients with schizophrenia: a 6-week randomized, doubleblind, placebo-controlled, multicenter study. Schizophr Res. 2019 (ePublished).

14. Isaac M, Holvey $\mathrm{C}$. Transdermal patches: the emerging mode of drug delivery system in psychiatry. Ther Adv Psychopharmacol. 2012;2(6):255-63.

15. Pharmaceuticals and Medical Devices Agency. New drug application review report for Lonasen tape $20 \mathrm{mg}, 30 \mathrm{mg}$ and $40 \mathrm{mg}$. CTD 2.5 Clinical Overview. 2019. http://www.pmda.go.jp/drugs /2019/P20190704001/index.html. Accessed 01 Nov 2019.

16. Kawamura T, Sasagawa S, Masuda T, Honda S, Kinoshita M, Harada $\mathrm{S}$, et al. Basic studies on the standardization of patch test. 1970;80(5):301-14.

17. Inada T, Beasley CM Jr, Tanaka Y, Walker DJ. Extrapyramidal symptom profiles assessed with the Drug-Induced Extrapyramidal Symptom Scale: comparison with Western scales in the clinical double-blind studies of schizophrenic patients treated with either olanzapine or haloperidol. Int Clin Psychopharmacol. 2003;18(1):39-48.

18. Posner K, Brown GK, Stanley B, Brent DA, Yershova KV, Oquendo MA, et al. The Columbia-Suicide Severity Rating Scale: initial validity and internal consistency findings from three multisite studies with adolescents and adults. Am J Psychiatry. 2011;168(12):1266-77.

19. Lindenmayer JP, Bernstein-Hyman R, Grochowski S. A new five factor model of schizophrenia. Psychiatr Q. 1994;65(4):299-322.

20. Hogan TP, Awad AG, Eastwood R. A self-report scale predictive of drug compliance in schizophrenics: reliability and discriminative validity. Psychol Med. 1983;13(1):177-83.

21. The EuroQOL Groups. EuroQol-a new facility for the measurement of health-related quality of life. Health Policy. 1990;16(3):199-208.

22. Ikeda S, Shiroiwa T, Igarashi A. Developing a Japanese version of the EQ-5D-5L value set. J Nat Inst Pub Health. 2015;64:47-55.

23. Gaebel W, Riesbeck M, von Wilmsdorff M, Burns T, Derks EM, Kahn RS, et al. Drug attitude as predictor for effectiveness in first-episode schizophrenia: results of an open randomized trial (EUFEST). Eur Neuropsychopharmacol. 2010;20(5):310-6.

24. Hirayasu YT, Iizumi M, Kikuchi H. A long-term study of paliperidone extended-release tablets in patients with schizophrenia. Jpn J Clin Psychopharmacol. 2010;13:2105-35.

25. Hishikawa Y, Kaneko S, Kondo T, Sakai A, Ueda H, Abe S, Imamura S, Tashiro T. Phase III long-term clinical study of aripiprazole- - a multicenter open-study to determine safety and effectiveness in schizophrenic patients (Aomori, Iwate, and Akita regions study group). Jpn J Clin Psychopharmacol. 2006;9:1211-35.

26. Kinoshita T, Waku M, Tamura F, Iwama Y. Long-term safety and efficacy of sublingual asenapine for the treatment of schizophrenia: a phase III extension study with follow-up for 52 weeks (P06125). Jpn J Clin Psychopharmacol. 2016;19:753-70.

27. Ishigooka J, Iwashita S, Tadori Y. Long-term safety and effectiveness of brexpiprazole in Japanese patients with schizophrenia: a 52-week, open-label study. Psychiatry Clin Neurosci. 2018;72(6):445-53.

28. National Collaborating Centre for Mental Health. National Institute for Health and Clinical Excellence: Guidance. Schizophrenia: core interventions in the treatment and management of schizophrenia in primary and secondary care (update). Leicester: British Psychological Society. 2009. 
29. Fenton WS, McGlashan TH. Natural history of schizophrenia subtypes. II. Positive and negative symptoms and long-term course. Arch Gen Psychiatry. 1991;48(11):978-86.

30. Cerveri G, Gesi C, Mencacci C. Pharmacological treatment of negative symptoms in schizophrenia: update and proposal of a clinical algorithm. Neuropsychiatr Dis Treat. 2019;15:1525-35.

31. Weiden PJ, Kozma C, Grogg A, Locklear J. Partial compliance and risk of rehospitalization among California Medicaid patients with schizophrenia. Psychiatr Serv. 2004;55(8):886-91.
32. Lewis DA, Lieberman JA. Catching up on schizophrenia: natural history and neurobiology. Neuron. 2000;28(2):325-34.

33. Hamann J, Cohen R, Leucht S, Busch R, Kissling W. Shared decision making and long-term outcome in schizophrenia treatment. J Clin Psychiatry. 2007;68(7):992-7.

34. Nielsen RE, Lindstrom E, Nielsen J, Levander S. DAI-10 is as good as DAI-30 in schizophrenia. Eur Neuropsychopharmacol. 2012;22(10):747-50.

\section{Affiliations}

\section{Nakao Iwata ${ }^{1}$ (D) Jun Ishigooka ${ }^{2}$ - Ichiro Naoi $^{3}$ (D) $\cdot$ Masahiro Matsumoto $^{3}$ (D) Yuichi Kanamori $^{3}$ (D) . Hiroshi Nakamura $^{3}$ (D) Teruhiko Higuchi ${ }^{4}$ D}

\author{
Hiroshi Nakamura \\ hiroshi-nakamura@ds-pharma.co.jp \\ Nakao Iwata \\ nakao@fujita-hu.ac.jp \\ Jun Ishigooka \\ ishigooka@i-cnsp.com \\ Teruhiko Higuchi \\ higuchi2914@yahoo.co.jp
}

1 Department of Psychiatry, Fujita Health University, 1-98, Dengakugakubo, Kutsukake-cho, Toyoake-shi, Aichi 470-1192, Japan

2 Institute of CNS Pharmacology, 4-26-11, Sendagaya, Shibuya-ku, Tokyo 151-0051, Japan

3 Sumitomo Dainippon Pharma Co., Ltd., 13-1, Kyobashi 1-Chome, Chuo-ku, Tokyo 104-8356, Japan

4 Japan Depression Center, 1-7, Rokubancho, Chiyoda-ku, Tokyo 102-0085, Japan 